\title{
A Linear Continuous Feedback Control of Chua's Circuit
}

\author{
CHI-CHUAN HWANG \\ Department of Mechanical Fngineering, Chung-Yuan Iniversity, Chung-I i, Taiwan 32023, ROC.
}

JIN-YUAN HSIEH

Department of Mechanical Engineering, Nan Yan Junior College, Chung-Li, Taiwan 32024, ROC

and

RONG-SYH LIN

Department of Mechanical Engineering, Chung-Yuan University, Chung-Li, Taiwan 32023, ROC

\begin{abstract}
A continuous feedback controller is designed to suppress and eliminate the chaotic behavior of Chua's circuit. This controller is constructed by the following two phases. First, the set of equilibrium points is extended by virtue of embedding suitable feedback terms in the control channel. Second, a state error term is then added to steer the dynamics of the control system to a fixed point or a limit cycle. The stability region of Chua's circuit with control is determined via the Routh-Hurwitz criterion. Some numerical simulations are given to demonstrate the effect of the control design. (C) 1997 Elsevier Science Ltd
\end{abstract}

\section{INTRODUCTION}

The detail dynamics in a chaotic system cannot be grasped accurately from instance to instance due to not only its complication but also its uncertainty. If chaos cannot be: controlled, it might on many occasions result in disaster and collapse a dynamical system. In recent years, after the pioneering work of Ott, Grebogi and Yorke (OGY) [1], controlling chaos has become more and more interesting in academic research and practical applications.

Generally speaking, there are two ways to control chaos: feedback control [1-3] and nonfeedback control $[4,5]$. The nonfeedback control is mainly suitable for occasions that require high-speed control such as fast electro-optical systems. On the other hand, however, due to the weak robustness and the computational arduousness that a nonfeedback control may encounter, the feedback control is therefore preferred in other applications. As to the feedback control, the OGY method is famous and is widely used by many other researchers. Dressler and Nitsche [3] introduced the so-called time-delay coordinates method by modifying the OGY method so that it can be applied in an experiment system. The OGY method was further modified or corrected in order to be suitable for other systems, giving, for example, the OPF method [6] and the RPF method [7] applied in high dissipative systems, the PPF method [8] applied in systems without adjustable parameters, and the MED method [9] applied in systems with complicated eigenvalues. In these methods, the technique of intermittent perturbations commonly used is quite inconvenient and it is sometimes very difficult to find the adjustable parameter for perturbation. To overcome these problems, the theory of continuous feedback control now has been researched and developed. 
Chua's circuit has been studied extensively as a prototypical electronic system [10]. Chen and Dong $[11,12]$ controlled the chaotic trajectory of the circuit to reach the limit cycle by using a linear feedback control. By applying the input-output and state-space techniques, Hartley and Mossayebi [13] showed the control of modified Chua s circuit systems and demonstrated how to design a controller for tracking the capacitor voltage of the system. Saito and Mitsubori [14] proposed a simple control method to stabilize chaotic attractor to desired periodic orbit. Ramirez [15] presented a nonlinear feedback control to a chaotic system. Unfortunately, their nonlinear feedback occasionally becomes singular, i.e. it is not controllable for singular points. By applying a similar method to that presented by Ramirez. Hwang et al. [16] proposed a feedback control on a modified Chua's circuit with cubic nonlinearity and have successfully controlled the chaotic dynamics to fixed points.

In this paper, we will follow Hwang et al. [16] but will focus on the Chua s circuit in which the nonlinearity will be closer to the real performance of the electronic components. Besides controlling the chaos to fixed points, in this paper we will further show the control of chaos to desired limit cycles.

\section{FORMULATION}

Chua 's circuit consists of one inductor, two capacitors, one linear resistor, and one piecewise-linear nonlinear resistor. The mathematical model equations [17] for this circuit are

$$
\begin{aligned}
& \dot{x}=\alpha(1+x-f(x)), \\
& \dot{y}=x-1+\therefore \\
& \therefore \therefore \beta
\end{aligned}
$$

wher:

$$
f(x)=\left\{\begin{array}{cc}
b x+a-b, & x-1 \\
a x & x y \leq 1 \\
b x-a+b, & x-1
\end{array}\right.
$$

In the model equations, variables $x$ and $y$ represent the voltages across the two capacitors, and variable $z$ in the current through the inductor. Typical values of the system parameters $(\alpha, \beta, a, b)$ are chosen to be $(9,100 / 7,-8 / 7,-5 / 7)$ so that chaotic behaviors can be found in the dynamical system (1). Through this choice, the corresponding equilibrium points of system (1) clearly are $(1.5,0,-1.5),(0,0,0)$, and $(-1.5,0,1.5)$, which happen to be located in the regions $x>=1,|x|<=1$, and $x<\cdots-1$, respectively. For the chaotic behavior of Chua's circuit to be controlled or suppressed. Hwang et al. [16] have suggested a method of feedback control by adding in series with the inductor a voltage source $u$, which is then the controller of the system. With this design. the whole system becomes

$$
\begin{aligned}
& x=\alpha(y-x-f(x)), \\
& i-x-z) \\
& \therefore-\beta y-4 .
\end{aligned}
$$

The controller $u$ has two feedbacks. The first one is designed so as to mimic the dynamics of the second equation of system (2), and consequently the equilibrium points of system (1) will be extended to an equilibrium manifold $(x, x+f(x), f(x))$. If given a desired state, say $\left(x_{\text {ret }}, x_{\text {ret }}+f\left(x_{\text {ret }}\right), f\left(x_{\text {ref }}\right)\right)$ to be tracked, then a proportional feedback $k_{\mathrm{p}}\left(x_{\text {ref }}-x\right)$ is added to be the second feedback of controller $u$. If the input state is $x$, then the controller $u$ is expressed by

$$
u=u_{1}+u_{n}=k\left(x+y+n+\beta v+k_{p}\left(x_{t, 1} \cdot x\right)\right.
$$




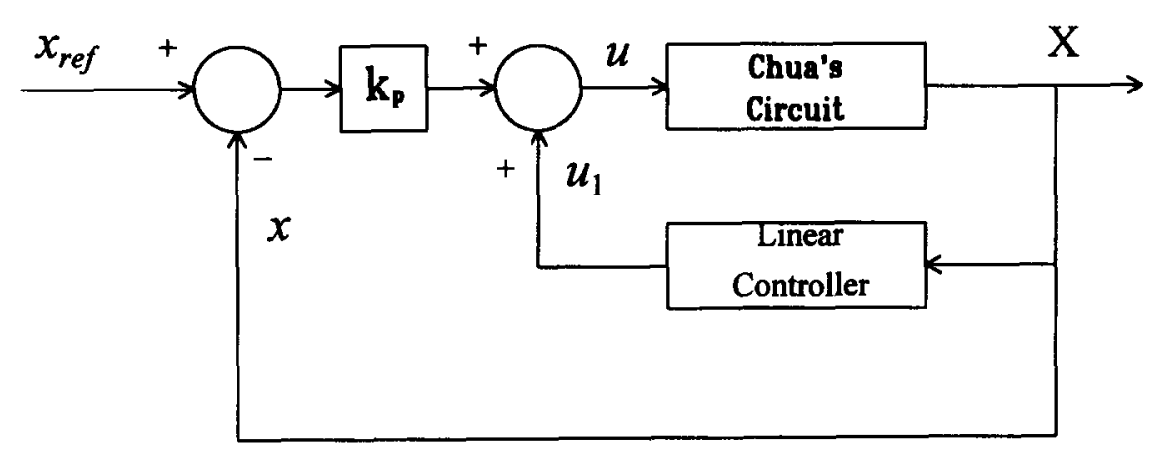

Fig. 1. The block diagram of a controlled system. The vector $\mathbf{X}$ is the set of all state variables.

If instead the input state is variable $y$ then the second feedback of the controller $u$ should be replaced by $u_{2}=k_{\mathrm{p}}\left(y_{\mathrm{ref}}-y\right)$. The block diagram of the control system is shown in Fig. 1 . The main advantages of such a design proved by Hwang et al. are that the present controller may provide a shorter settling time with lower overshoot than other controllers, and it can eliminate the tracking error without using another integral controller.

If the input state is $x$, then the corresponding Jacobian and its characteristic equation with respect to the general equilibrium manifold are, respectively,

$$
\mathbf{J}=\left[\begin{array}{ccc}
-\alpha-\alpha b & \alpha & 0 \\
1 & -1 & 1 \\
\mathbf{k}-\mathbf{k}_{\mathrm{p}} & -k & k
\end{array}\right]
$$

for $|x|>1$, and

$$
|\lambda I-J|=\lambda^{3}+(1-k+\alpha+\alpha b) \lambda^{2}+\alpha(b-k-b k) \lambda+\alpha k_{\mathrm{p}}=0 .
$$

With the characteristic equation (4), one can justify the stability of the equilibrium manifold by applying the Routh-Hurwitz criterion. Noting that the solution of eigenvalue $\lambda$ of the Jacobian is determined by the values of the gains $k$ and $k_{\mathrm{p}}$, with the other parameters fixed, we can thus plot a borderline dividing the regions of stability in the $k-k_{\mathrm{p}}$ plane, as shown in Fig. 2(a). So, if the chaos is to be controlled to a fixed point, the choice of $\left(k, k_{\mathrm{p}}\right)$ should be taken from the stable region shown in Fig. 2(a). In the following numerical experiments, we have done this. Likewise, without showing the detailed derivation, we have also plotted the stability borderline in the $k-k_{\mathrm{p}}$ plane in Fig. 2(b) where the $y$-variable is chosen to be the input state.

\section{NUMERICAL EXPERIMENTS}

In this section, we will show a series of numerical experiments by using the fourth-order Runge-Kutta method with step size 0.01 . The initial condition $x=0.15264, y=-0.0228 \mathrm{l}$, $z=0.54467$, is chosen in all the simulations so that Chua's circuit will exhibit chaotic behaviors if no control is applied [17]. The performance of the present control will not be emphasized here because its excellence has been shown by being compared with those of other control in the previous paper of Hwang et al. [16].

\subsection{Controlling the chaos to fixed points}

In the first numerical experiment, we intend to control the chaos to the equilibrium point $(1.5,0,-1.5)$ of system (1), i.e. by setting $x_{\mathrm{ref}}=1.5$. The control is activated at time $t=40$. Figure 3(a) shows the successful suppression to the chaotic behavior. Other than the 

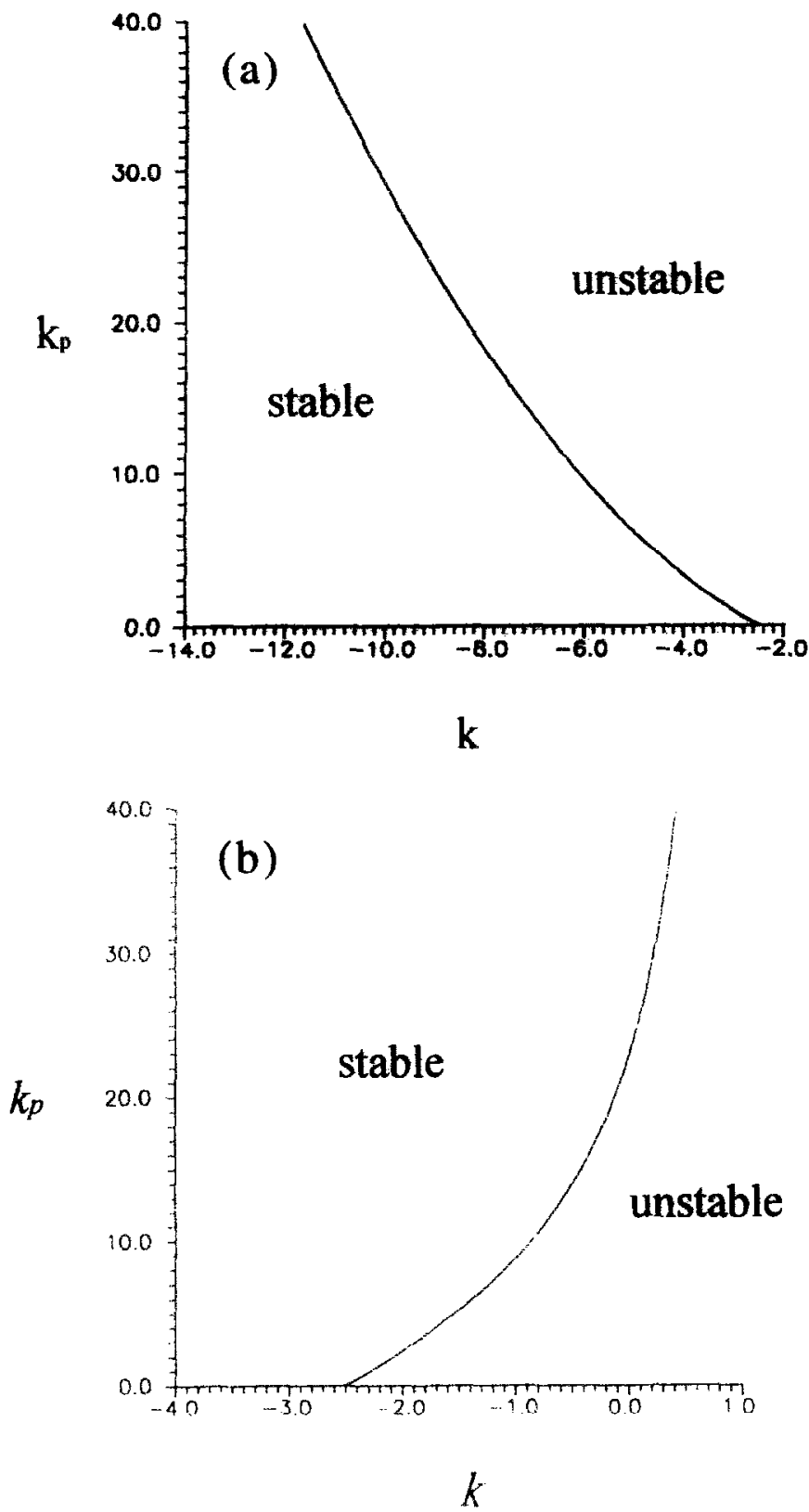

Fie. 2. (a) The stability diagram in the $k-k_{p}$ plane using $x$ as the control input. (b) The stability diagram in the $k-k_{,}$plane using $k$ as the control input.

equilibrium point of the uncontrolled system (1), the chaos can also be suppressed to any desired fixed point on the equilibrium manifold. For example, we have set $x_{\text {ref }}=-4$ in the second simulation. The dynamical evolution of variable $x$ is shown in Fig. 3(b). In this case. the control is also activated at the time $t=40$.

We can also choose the $y$-variable as the input state. If we do so, then the associated Jacobian and its characteristic equation will be different from experiments (4) and (5), but we are not intending to show them. Similarly. in the following numerical experiments, the 

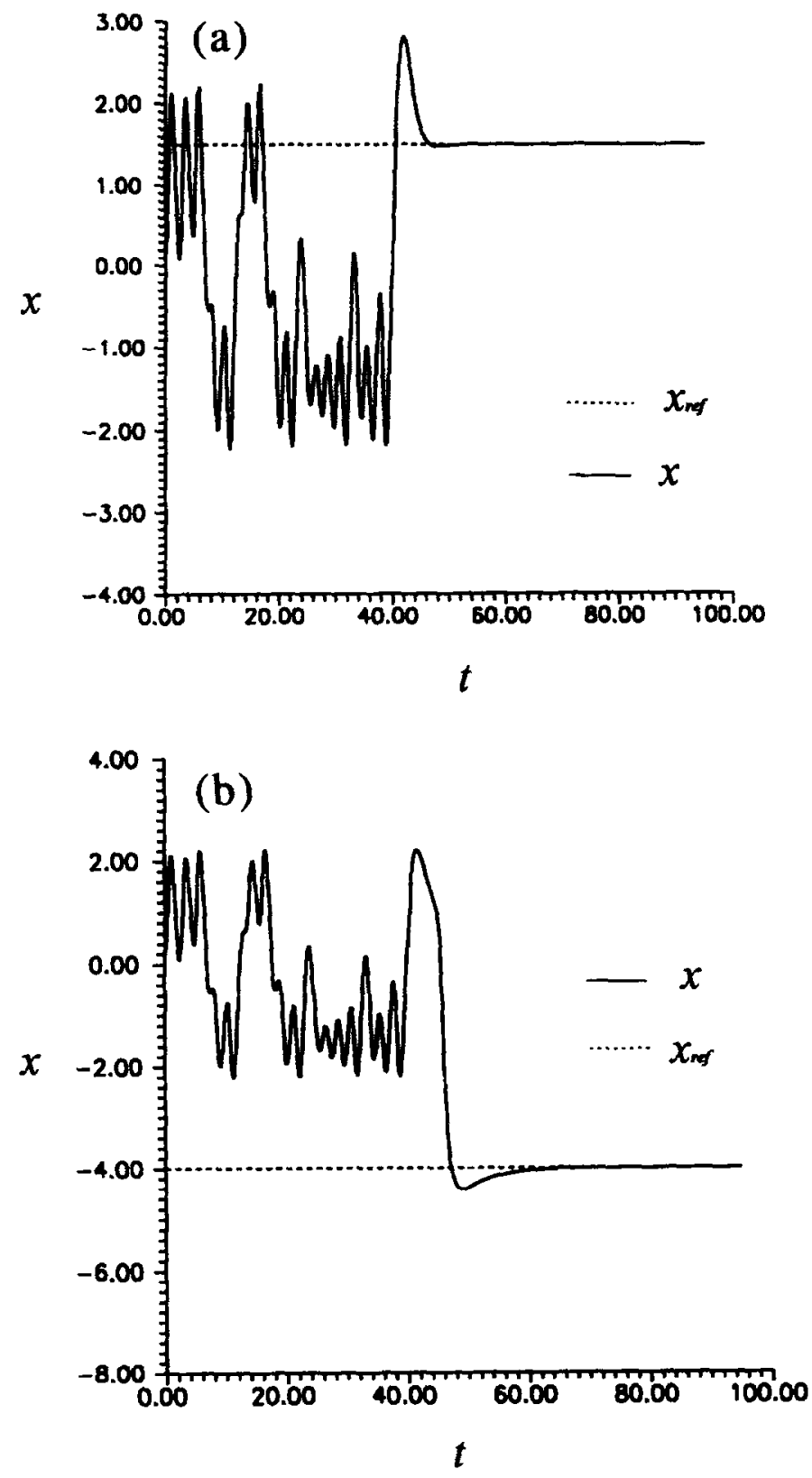

Fig. 3. (a) The time responses of the controlled system, $x_{\text {ref }}=1.5$. (b) The time responses of the controlled system, $x_{\text {vel }}--4$. The control $u$ is activated at $t=40$.

valves of $k$ and $k_{\mathrm{p}}$ should be taken from the stable region shown in Fig. 2(b). In the third case, we set $y_{\text {ref }}=0$, which corresponds to the equilibrium point of the uncontrolled system (1). The control is activated at the time $t=40$. We shown the dynamical development of variable $y$ in Fig. 4(a). For showing the control of chaos to another fixed point on the equilibrium manifold, we set $y_{\text {ref }}=4$ in the fourth simulation. The activating time is again at $t=40$. Figure $4(\mathrm{~b})$ shows the successful result under the application of the present control. 

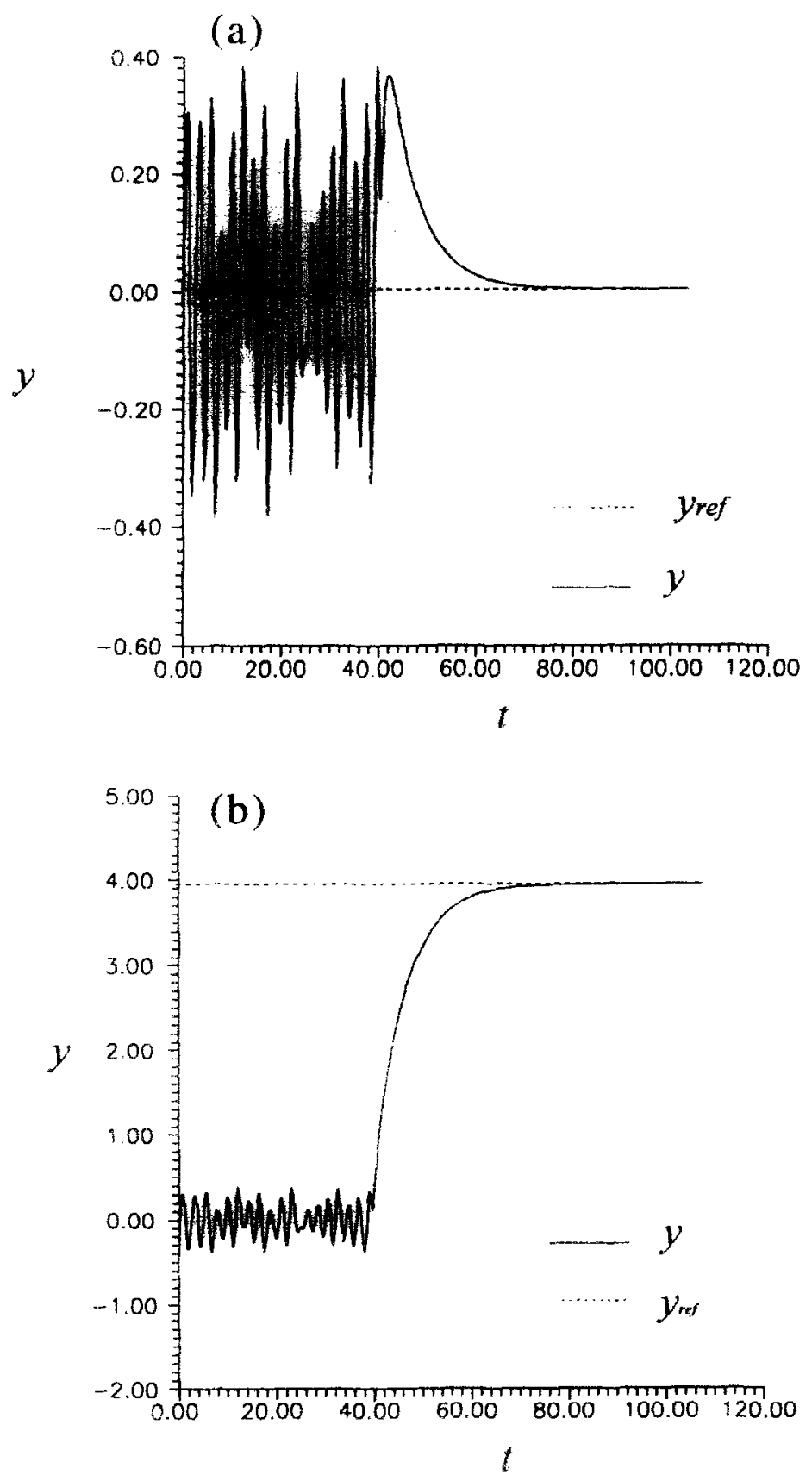

Fis. 4. (a) The time responses of the controlled system, $y_{\text {iet }}=0$. (h) The time responses of the controlled system. .. - f. The contral $u$ is activated at: - 4 !

\subsection{Controlling chaos to limit cycles}

So far we have shown that the chaotic behaviors of Chua's circuit can be suppressed to a desired fixed point on the equilibrium manifold. By applying the control system proposed by Hwang et al., as the composition of $\left(k, k_{\mathrm{p}}\right)$ is chosen from the stability regions shown in Fig. $2(a)$ and (b). Besides this, controlling chaos to a desired limit cycle is also an important task for the relevant researchers. In the following, we will show that the present control can also control the chaos to a period-1 orbit successfully. 

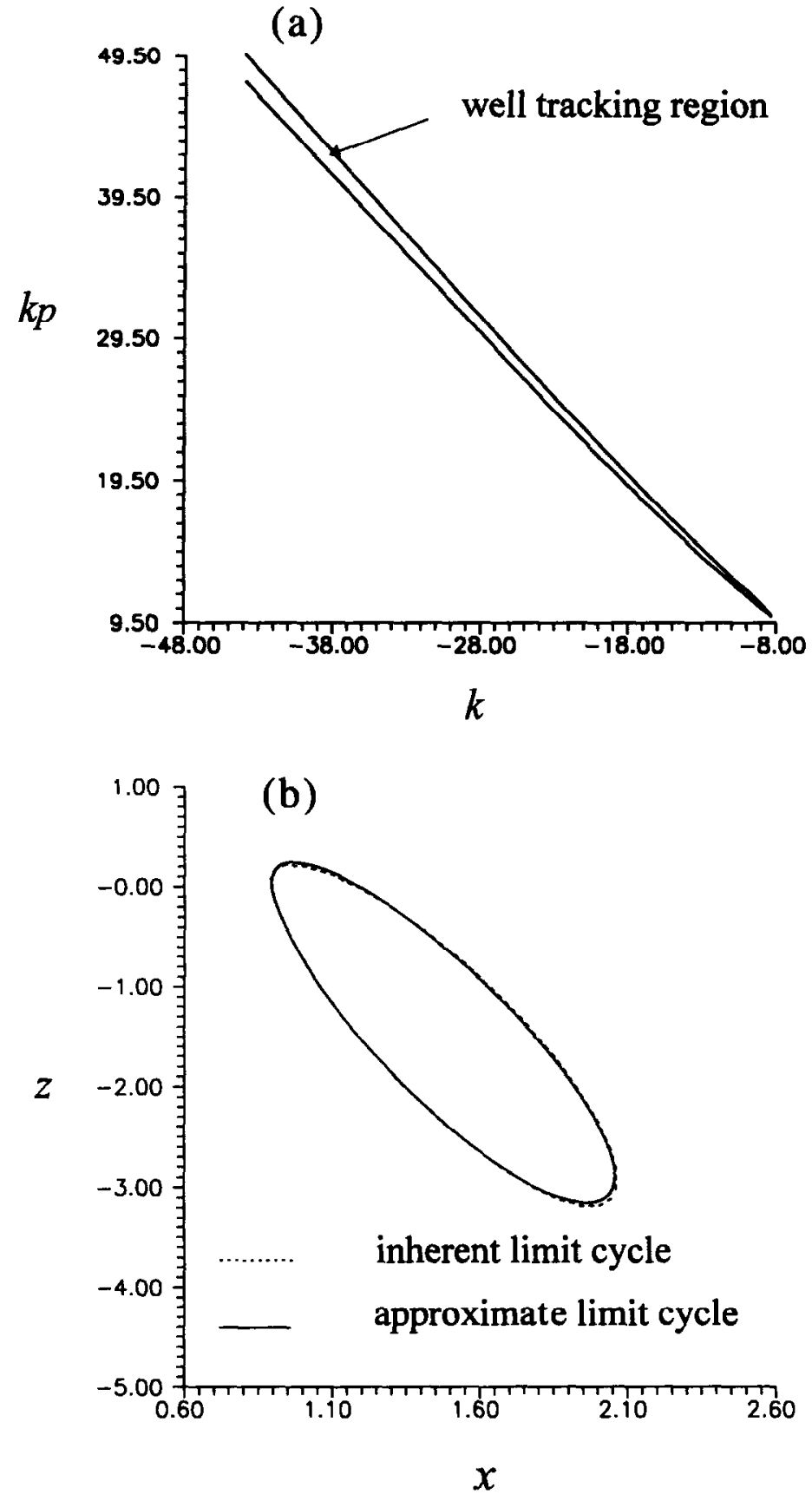

Fig. 5. (a) The well tracking region for a limit cycle in the $k-k_{\mathrm{p}}$ plane. (b) The $x-z$ phase plane with $k_{\mathrm{p}}=19.73$. $k=-18$. The control $u$ is activated at $t=17$.

Theoretically, with the present controller, the whole system (2) may have a period-1 solution of the point $\left(k, k_{\mathrm{p}}\right)$ and is chosen to be on the stability borderline. Actually, during the tracking process, the control parameters $\left(k, k_{\mathrm{p}}\right)$ should be taken from an area instead of a line for allowance of error. For example, Fig. 5(a) shows in the $k-k_{\mathrm{p}}$ plane the best control 
parameter area for the steady-state error within $5 \%$. Figure 5(b) shows in the $x-z$ phase plane the corresponding limit cycle, the solid circle, for $\left(k, k_{\mathrm{p}}\right)=(-18.0,19.73)$ from this area, while in the same figure the broken circle represents the theoretical limit cycle. In the last numerical experiment of this paper, when the controller is activated at the same time $t=17$, the $x$-variable is transformed from chaos to a periodic trajectory and its time-wave diagram is plotted in Fig. 6(a). The corresponding dynamical development of the controller $u$
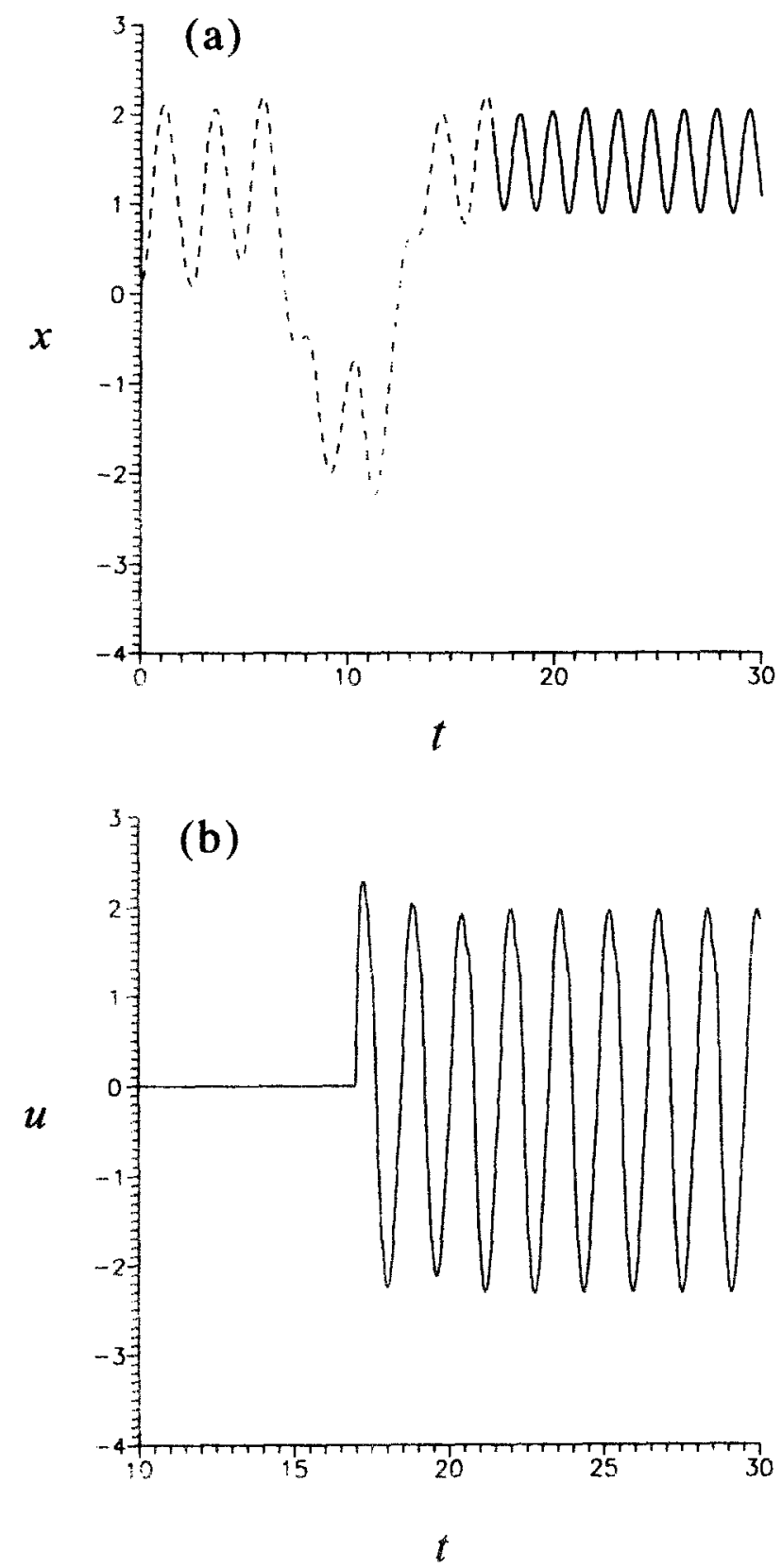

Fig. 6. (a) The time responses of the controlled system for tracking a limit cycle with $k_{\mathrm{p}}=19.73, k=-18$. (b) The control action vs time. The control $u$ is activated at $t=17$. 
is shown in Fig. 6(b). From these figures, we can see that the same advantage of the present design for controlling chaos to fixed points can also be found in controlling chaos to limit cycles, i.e. the shot settling time and small overshoot.

\section{CONCLUSIONS}

Chaotic behaviors can be found in Chua's circuit. The chaos, however, can be suppressed by adding to the system a feedback controller proposed by Hwang et. al. [16]. In this paper, we adapt the piecewise-linear model for the nonlinear resistor in the circuit system, instead of the cubic nonlinearity model adapted in the work of Hwang et al. Given the input state, the corresponding Jacobian and its characteristic equation of the system can be derived. The stability criterion to the characteristic equation can help decide the choice of the control parameters for controlling the chaos. The main advantages of the present control, including shot settling time and small overshoot, not only can be found in controlling chaos to fixed points but also, after the least simulation of this paper, can be found in controlling chaos to limit cycles.

\section{REFERENCES}

1. Ott, E., Grebogi, C. and Yorke, J. A., Controlling chaos. Phys. Rev. Lett., 1990, 64, 1196-1199.

2. Singer, J., Wang, Y. Z. and Bau, H. H., Controlling a chaotic system. Phys, Rev. Lett., 1991, 66, 1123-1125.

3. Dressler, U. and Nitsche, G., Controlling chaos using time delay coordinates. Phys. Rev. Lett., 1992, 68, 1-4.

4. Braiman, Y. and Goldhirsch, I., Taming chaotic dynamics with weak periodic perturbations. Phys. Rev. Lett., $1991,66,2545-2548$.

5. Lima, R. and Pettini, M., Suppression of chaos by resonant parametric perturbations. Phys. Rev. A, 1990, 41, 726-733.

6. Petrov, V., Gaspar, V., Masere, J. and Showalter, K., Controlling chaos in the Belousov-Zhabotinsky reaction. Nature, 1993, 361, 240-243.

7. Parmananda, P., Sherard, P., Rollins, R. W. and Dewald. H. D., Control of chaos in an electrochemical cell. Phys. Rev. E, 1993, 47, 3003-3006.

8. Garfinkel, A., Spano, M. L., Ditto, W. L. and Weiss, J. N., Controlling cardiac chaos. Science, 1992, 257, $1230-1235$.

9. Reyl, C., Flepp, L., Badii, R. and Brun, E., Control of NMR-laser chaos in high-dimensional embedding space. Phys. Rev. E, 1993, 47, 267-272.

10. Madan, R. N. (ed.), Chua's Circuit: A Paradigm for Chaos. World Scientific, Singapore, 1993.

11. Chen, G., Controlling chaotic trajectories to unstable limit cycles-a case study. In IEEE Proc. of Amer. Contr. Conf., San Francisco, CA, June 1993, pp. 2413-2414.

12. Chen, G., Controlling Chua's global unfolding circuit family. IEEE Trans. Circuits Systems, 1993, 40, 829 -832.

13. Hartley, T. T. and Mossayebi, F., Control of Chua's circuit. J. Circuits Systems Comput., 1993, 3, $173-194$.

14. Saito, T. and Mitsubori, K., Control of chaos from a piecewise linear hysteresis circuit. IEEE Trans. Circuits Systems, 1995, 42, 168-172.

15. Ramirez, J. A., Nonlinear feedback for controlling the Lorenz equation. Phys. Rev. E, 1994, 50, $2239-2342$.

16. Hwang, C. C.. Chow, H. Y. and Wang, Y. K., A new feedback control of a modified Chua's circuit system. Phys. D, 1996, 92, 95-100.

17. Matsumoto, T., Chua, L. O. and Komuro, M., The double scroll. IEEE Trans. Circuits Systems, 1985,32, $798-818$. 